\title{
OUT-OF-PLANE MOTION ABOUT LIBRATION POINTS: NONLINEARITY AND ECCENTRICITY EFFECTS
}

\author{
T. A. HEPPENHEIMER* \\ Science Applications, Inc., Schiller Park, Ill., U.S.A.
}

(Received 30 November, 1971)

\begin{abstract}
Out-of-plane motion about libration points is studied within the framework of the elliptic restricted three-body problem. Nonlinear motion in the circular restricted problem is given to third order in the out-of-plane amplitude $A_{z}$ by Jacobi elliptic functions. Linear motion in the elliptic problem is studied using Mathieu's and Hill's equations. Additional terms needed for a complete third-order theory are found using Lindsted's method. This theory is constructed for the case of collinear libration points; for the case of triangular points, a third-order nonlinear solution is given separately in terms of Jacobi elliptic functions.
\end{abstract}

\section{Introduction}

In the restricted three-body problem, the topic of in-plane motion about libration points has received exhaustive attention. With the exceptions of Moulton (1920), and Farquhar and Kamel (1972), however, most investigators have paid only cursory attention to the out-of-plane motion, beyond noting that under the linearized equations of the restricted three-body problem, this motion is simple harmonic.

The present author (Heppenheimer, 1970), studying the prevention of occultation by the Moon of a libration-point satellite, found that the period of the out-of-plane motion must be accurately known for the occultation-prevention method not to cause a secular perturbation in the out-of-plane amplitude. There thus are practical reasons for studying the out-of-plane motion in detail.

Accordingly, this paper first considers nonlinear motion in the circular restricted three-body problem, then linear motion in the elliptic problem. By combining these solutions, most of the necessary terms for a nonlinear-elliptic solution are at hand; the remaining terms are computed separately. Perturbation parameters are the out-ofplane amplitude $A_{z}$ and eccentricity $e$ of the primaries; solutions are carried to third order in these quantities. These solutions are given for the case of collinear points; the case of triangular points is treated separately.

Under the usual normalization of units in the circular restricted problem, the equations of motion are (Szebehely, 1967, pp. 558 and 594):

$$
\begin{aligned}
& \ddot{x}-2 \dot{y}=\Omega_{x} \\
& \ddot{y}+2 \dot{x}=\Omega_{y} \\
& \ddot{z}+z=\Omega_{z}
\end{aligned}
$$

* Work performed as a National Science Foundation Graduate Fellow, Department of Aerospace Engineering, University of Michigan, Ann Arbor, Michigan 48104. 
and

and

$$
\Omega=\frac{1}{2}\left(x^{2}+y^{2}+z^{2}\right)+\frac{1-\mu}{r_{1}}+\frac{\mu}{r_{2}}+\frac{1}{2} \mu(1-\mu)
$$

$$
r_{1}^{2}=(x-\mu)^{2}+y^{2}+z^{2} ; \quad r_{2}^{2}=(x+1-\mu)^{2}+y^{2}+z^{2}
$$

and $\mu,(1-\mu)$ are the normalized masses of the smaller and larger primaries respectively. In the circular problem, distances are normalized with respect to the constant distance between primaries, and their mean anomaly is used as the independent variable. In the elliptic restricted problem, distances are normalized with respect to the variable distance between primaries, and true anomaly is used as the independent variable. The equations of motion then show formal similarity to (1) and (3) but not to (2):

$$
\begin{aligned}
& x^{\prime \prime}-2 y^{\prime}=\bar{\Omega}_{x} \\
& y^{\prime \prime}+2 x^{\prime}=\bar{\Omega}_{y} \\
& z^{\prime \prime}+z=\bar{\Omega}_{z}
\end{aligned}
$$

and

and

$$
\bar{\Omega}=(1+e \cos \theta)^{-1}\left[\frac{1}{2}\left(x^{2}+y^{2}+z^{2}\right)+\frac{1-\mu}{r_{1}}+\frac{\mu}{r_{2}}+\frac{1}{2} \mu(1-\mu)\right]
$$

$$
r_{1}^{2}=(x-\mu)^{2}+y^{2}+z^{2} ; \quad r_{2}^{2}=(x+1-\mu)^{2}+y^{2}+z^{2}
$$

and $\theta$ is true anomaly. Note that ()$^{\prime}=\mathrm{d}(\quad) / \mathrm{d} \theta$.

It is a consequence of this formal similarity that the positions of the libration points, in the coordinate system, are not functions of $e$ but remain, as in the circular problem, only functions of $\mu$. This has important consequences for the construction of a thirdorder theory for out-of-plane motion about libration points. Such a theory will contain no terms of orders $e, e^{2}, e^{3}$, etc. The only terms that will appear are as follows: First order: $A_{z}$

Second order: $A_{z}^{2}, e A_{z}$ Third order: $A_{z}^{3}, e A_{z}^{2}, e^{2} A_{z}$

A nonlinear-circular case solution will contribute terms of order $A_{z}, A_{z}^{2}, A_{z}^{3}$; a linear-elliptic case solution will contribute terms of order $A_{z}, e A_{z}, e^{2} A_{z}$. Thus only the terms of order $e A_{z}^{2}$ must be computed separately.

To begin, consider the third of Equations (1). Let us consider a collinear libration point; the case of the triangular points will be noted separately. Linearizing about that point, considered to lie at $\left(x=x_{0}, y=0, z=0\right)$ :

$$
\ddot{z}+A z=0
$$

where

$$
A=(1-\mu)\left(x_{0}-\mu\right)^{-3}+\mu\left(x_{0}+1-\mu\right)^{-3}
$$

so the solution may be written,

$$
z=A_{z} \sin \sqrt{ } \bar{A}\left(t-t_{0}\right)
$$




\section{Circular Problem - Nonlinear Motion}

Consider the first two of Equations (1), in which the right-hand sides $\Omega_{x}, \Omega_{y}$ are expanded about the point $\left(x_{0}, 0,0\right)$ as power series in $x, y, z$, the expansions being taken to terms of second order:

$$
\begin{aligned}
& \ddot{x}-2 \dot{y}=(1+2 A) x+\frac{3}{2} B\left(-2 x^{2}+y^{2}+z^{2}\right)+\cdots \\
& \ddot{y}+2 \dot{x}=(1-A) y+3 B x y+\cdots
\end{aligned}
$$

Also, the third of Equations (1) is expanded out to third-order terms:

$$
\ddot{z}+A z=3 B x z+\frac{3}{2} C\left(-4 x^{2} z+y^{2} z+z^{3}\right)+\cdots
$$

The term $A$ is given by Equation (8); the terms $B, C$ are

$$
\begin{aligned}
& B= \pm(1-\mu)\left(x_{0}-\mu\right)^{-4} \pm \mu\left(x_{0}+1-\mu\right)^{-4} \\
& C=(1-\mu)\left(x_{0}-\mu\right)^{-5}+\mu\left(x_{0}+1-\mu\right)^{-5}
\end{aligned}
$$

and in defining $B$, the upper, middle, or lower signs are used depending on whether the collinear libration point under consideration is $L_{2}, L_{1}$, or $L_{3}$, respectively. [ $L_{1}$ lies between the primaries, $L_{2}$ is near the mass $\mu, L_{3}$ near the mass $(1-\mu)$.]

Now assume $z \gg x, y$, an assumption justified by the nonlinear coupling of $z$ to $x, y$. Then terms in $x^{2}, x y, y^{2}$ are to be neglected in comparison with terms in $z^{2}$, and Equations (10) become a linear system with forcing function:

$$
\begin{aligned}
& \ddot{x}-2 \dot{y}-(1+2 A) x=\frac{3}{2} B z^{2} \\
& \ddot{y}+2 \dot{x}-(1-A) y=0
\end{aligned}
$$

and $z=z(t)$ is given by Equation (9). The forced motion corresponding to this input then is given as

$$
\begin{aligned}
& x=-\frac{3}{4} B A_{z}^{2}\left[\frac{1}{1+2 A}-\frac{1+3 A}{1-7 A+18 A^{2}} \cos 2 \sqrt{A} t\right] \\
& y=-3 B \sqrt{A} A_{z}^{2}\left[\frac{\sin 2 \sqrt{A} t}{1-7 A+18 A^{2}}\right]
\end{aligned}
$$

Comparison with Equation (9) shows that the first of Equations (14) may be given explicitly in terms of $z$;

$$
x=\frac{-B}{1-7 A+18 A^{2}}\left[\frac{9 A(A-1) A_{z}^{2}}{1+2 A}+\frac{3}{2}(1+3 A) z^{2}\right]
$$

correct at least to order $A_{z}^{2}$. In fact, a continuation of the expansions of Equation (10) would give terms of order $A_{z}^{4}$ and higher; thus, Equation (15) is correct to order $A_{z}^{3}$. Note that $z=O\left(A_{z}\right), x=O\left(A_{z}^{2}\right)$.

Now consider Equation (11). The terms $x z, z^{3}$ are of order $A_{z}^{3}$; the terms $x^{2} z, y^{2} z$ 
are of order $A_{z}^{5}$ and are therefore to be neglected. Combining Equations $(11,15)$ then gives the third-order equation for $z=z(t)$ :

where

$$
\ddot{z}+u z-\frac{3}{2} v z^{3}=0
$$

and

$$
u=A+3 B \alpha ; \quad v=C-2 B \beta
$$

$$
\alpha=\frac{9 A B(A-1) A_{z}^{2}}{(1+2 A)\left(1-7 A+18 A^{2}\right)} ; \quad \beta=\frac{3 B(1+3 A)}{2\left(1-7 A+18 A^{2}\right)}
$$

Equation (16) possesses an integral, denoted $I$ :

$$
\dot{z}^{2}+u z^{2}-\frac{3}{4} v z^{4}=I
$$

so that $I, A_{z}$ are related by

$$
I=u A_{z}^{2}-\frac{3}{4} v A_{z}^{4}
$$

since the maximum value of $z$ is $z=A_{z}$.

Now we note that Equation (16) possesses solutions which are periodic in time; this follows from the fact that any one-dimensional system possessing a first integral will admit of periodic solutions (Goldstein, 1965, pp. 288-90).

From (19), the differential equation to be solved is then given by a quadrature:

$$
d t=\frac{\mathrm{d} z}{\sqrt{I-u z^{2}+\frac{3}{4} v z^{4}}}
$$

which can be reduced to Legendre's normal form of the elliptic integral of the first kind (Mathews and Walker, 1964, pp. 73-75) through the transformation

where

$$
\zeta=z \sqrt{ } n, \quad k^{2}=m / n
$$

$$
m=\frac{u}{2 I}\left[1-\sqrt{1-3 v I / u^{2}}\right], \quad n=\frac{u}{2 I}\left[1+\sqrt{1-3 v I / u^{2}}\right]
$$

which is obtained from (21) by writing

$$
1-\frac{u}{I} z^{2}+\frac{3 v}{4} \frac{v}{I} z^{4}=\left(1-m z^{2}\right)\left(1-n z^{2}\right)
$$

Then (21) becomes

$$
\mathrm{d} t=\frac{1}{\sqrt{I n}} \frac{\mathrm{d} \zeta}{\sqrt{\left(1-\zeta^{2}\right)\left(1-k^{2} \zeta^{2}\right)}}
$$

which is Legendre's normal form. Integrating and inverting the resultant elliptic integral to give an elliptic function, and making use of Equations $(22,23)$, gives $z=z(t)$ :

$$
z=\sqrt{\frac{2 I}{u\left[1+\sqrt{1-3 v I / u^{2}}\right]}} \times
$$




$$
\operatorname{sn}\left[t \sqrt{\frac{u}{2}\left[1+\sqrt{1-\frac{3 v I}{u^{2}}}\right]}, \sqrt{\frac{1-\sqrt{1-3 v I / u^{2}}}{1+\sqrt{1-3 v I / u^{2}}}}\right]
$$

which is the solution to (16). The complex expressions given as the argument and modulus of the elliptic function may be simplified in view of the required third-order accuracy. Thus, using (20),

$$
z=A_{z} \text { sn }\left[t \sqrt{u}\left[1-\frac{3}{8} \frac{v I}{u^{2}}\right], \sqrt{\frac{3}{4} \frac{v I}{u^{2}}}\right]
$$

or, for simplicity,

$$
z=A_{z} \operatorname{sn}(w t, k)
$$

It now is necessary to determine: (1) the effect of amplitude upon the period; (2) the formal expansion of (27) as a Fourier series. These results will be required for later solutions.

Amplitude effect on period: In consideration of Equation (28), the period is given by (Davis, 1962, p. 133)

$$
P=\frac{2 \pi}{w}\left[1+\frac{1}{4} k^{2}+O\left(k^{4}\right)\right]
$$

which is written,

$$
P=\frac{2 \pi}{\sqrt{A}}\left[\frac{A / u}{1-\frac{3}{4} v I / u^{2}}\right]^{1 / 2}\left[1+\frac{3}{16} \frac{v I}{u^{2}}\right]
$$

and, to third order, using $(17,18)$ :

$$
\sqrt{A / u}=1-\frac{3}{2} \frac{B}{A} \alpha
$$

so that, combining $(29,30)$,

$$
P=\frac{2 \pi}{\sqrt{A}}\left[1+A_{z}^{2}\left(\frac{9}{16} \frac{v}{u}-\frac{3}{2} \frac{B}{A} \frac{\alpha}{A_{z}^{2}}\right)\right]
$$

and, to third order,

$$
P=\frac{2 \pi}{\sqrt{ } \bar{A}}\left\{1+A_{z}^{2} \cdot \frac{9}{16 A}\left[C-3 B^{2}\left(\frac{1-3 A+14 A^{2}}{(1+2 A)\left(1-7 A+18 A^{2}\right)}\right)\right]\right\}
$$

and Equation (32) has been given by Moulton (1920).

Fourier-series expansion: Jacobi* has given an expansion of the elliptic function (see Whittaker and Watson (1963), p. 510):

$$
\operatorname{sn}(u, k)=\frac{2 \pi q^{1 / 2}}{K k}\left(\frac{\sin x}{1-q}+q \frac{\sin 3 x}{1-q^{3}}+q^{2} \frac{\sin 5 x}{1-q^{5}}+\cdots\right)
$$

* In his 1836 work on elliptic functions, written in Latin and titled Fundamenta Nova Functionarum Ellipticarum, usually called the Fundamenta Nova. 
where $u, x$ are dummy variables. We have

and

$$
u=2 K x / \pi ; \quad q=e^{-\pi K^{\prime} / K}
$$

$$
K=\frac{\pi}{2}\left[1+\frac{1}{4} k^{2}+\cdots\right] ; \quad K^{\prime}=\ln \left(\frac{4}{k}\right)+\frac{1}{4} k^{2}\left(\ln \left(\frac{4}{k}\right)-1\right)+\cdots
$$

so that (34) becomes, to third order,

$$
u=x\left(1+\frac{1}{4} k^{2}\right) ; \quad q=(k / 4)^{2} e^{1 / 2 k^{2}}
$$

so we have, $x=(2 \pi / P) t, P$ given by Equation (32). Then, using Equations (33-36), together with $(27,28)$ :

$$
\begin{aligned}
z= & A_{z} \sin (2 \pi / P) t+\frac{3}{64 A} A_{z}^{3}\left[C-\frac{3 B^{2}(1+3 A)}{1-7 A+18 A^{2}}\right][\sin 3(2 \pi / P) t+ \\
& +\sin (2 \pi / P) t]+O\left(A_{z}^{5}\right)
\end{aligned}
$$

The solution due to Moulton involves initial conditions selected so that the amplitude $A_{z}$ is unchanged due to incorporation of the nonlinear terms in (37); the resulting solution gives for the terms in the second bracket, $[\sin 3(2 \pi / P) t-3 \sin (2 \pi / P) t]$. The present solution involves no such special initial conditions. When this initialconditions effect is allowed for, Equation (37) becomes identical with the solution of Moulton.

Before leaving the topic of non-linear out-of-plane motion, it may be noted that the same type of elliptic-function solution may be found for the case of triangular libration points. For that case, Equations (8) and (12) are modified; thus, $\left(x_{0}-\mu\right)$ is replaced by $\left[\left(x_{0}-\mu\right)^{2}+y_{0}^{2}\right]^{1 / 2}$ and $\left(x_{0}+1-\mu\right)$ by $\left(\left[x_{0}+1-\mu\right)^{2}+y_{0}^{2}\right]^{1 / 2}$, the libration point being at $\left(x_{0}, y_{0}, 0\right)=\left[\frac{1}{2}-\mu, \pm \frac{1}{2} \sqrt{ } 3,0\right]$. Equations of motion analogous to (10, 11) have been given by Buck (1920), so the solution may proceed apace. It is given subsequently in this paper.

\section{Elliptic Problem - Linear Motion}

Having given the solution for nonlinear motion in the circular problem, we now consider linear motion in the elliptic problem. For the case of linear motion, the three equations of motion, Equations (4), uncouple so that only the third of Equations (4) need be considered. We have

$$
z^{\prime \prime}+z(1+e \cos \theta)^{-1}\left[e \cos \theta+\frac{1-\mu}{r_{1}^{3}}+\frac{\mu}{r_{2}^{3}}\right]=0 .
$$

Equation (38) is exact; it is linearized by considering $r_{1}, r_{2}$ as constant. Thus, applying Equation (8) and expanding in powers of $e$,

$$
z^{\prime \prime}+z\left[A-(A-1)\left(e \cos \theta-e^{2} \cos ^{2} \theta+e^{3} \cos ^{3} \theta-\cdots\right)\right]=0 .
$$

Equation (39) may be written as a Hill equation: see Whittaker and Watson (1963, 
p. 406). Define a new independent variable, $\phi: \phi=\theta / 2$. Then, replacing the powers of $\cos \theta$ by their finite Fourier-series equivalents, (39) becomes

$$
\frac{\mathrm{d}^{2} z}{\mathrm{~d} \phi^{2}}+z\left[a_{0}+2\left(a_{2} \cos 2 \phi+a_{4} \cos 4 \phi+a_{6} \cos 6 \phi+\cdots\right)\right]=0
$$

where

$$
\begin{aligned}
& a_{0}=4 A+(A-1)\left[2 e^{2}+\frac{3}{2} e^{4}+O\left(e^{6}\right)\right] \\
& a_{2}=-2(A-1)\left[e+\frac{3}{4} e^{3}+O\left(e^{5}\right)\right] \\
& a_{4}=(A-1)\left[e^{2}+e^{4}+O\left(e^{6}\right)\right] \\
& a_{6}=-(A-1)\left[\frac{1}{2} e^{3}+O\left(e^{5}\right)\right]
\end{aligned}
$$

The presence of the coefficients $a_{2 \mathrm{i}}(i=1,2,3, \ldots, \infty)$, each coefficient given as an infinite series in $e$, leads to an expression for the frequency involving a double infinity of terms, the celebrated Hill's infinite determinant. In accordance with known formulas (see McLachlan, 1947, pp. 127-9) the frequency, $v$, is found from

where

$$
\sin ^{2}\left(\frac{1}{2} \pi v\right)=\Delta \sin ^{2}\left(\frac{1}{2} \pi \sqrt{a_{0}}\right)
$$

$$
\Delta=1+\frac{\pi \cot \left(\frac{1}{2} \pi a_{0}^{2}\right)}{4 a_{0}^{2}}\left[\frac{a_{2}^{2}}{1^{2}-a_{0}}+\frac{a_{4}^{2}}{2^{2}-a_{0}}+\frac{a_{6}^{2}}{3^{2}-a_{0}}+\cdots\right]
$$

Equation (42) involves the first (and higher) powers of $a_{0}$ only; $a_{2}, a_{4}$, etc. appear squared. Thus, $v$ can involve only even powers of $e$, i.e. the eccentricity effect upon the frequency (or period) is an even function of $e$.

This result can be derived on physical grounds, since in the governing equations of motion, Equations (4-6), replacement of $e$ by $(-e)$ physically represents interchange of the apses of the orbit of the primaries; $\theta$ is measured not from pericenter but from apocenter. This change, however, is purely formal and cannot affect the period of motion; consequently the period must be an even function of $e$.

Thus, an expression for the period, correct to order $e^{3}$, will involve terms only of orders unity and $e^{2}$. The latter term may be found in terms of $a_{0}, a_{2}$ only; thus Equation (40) may be truncated and replaced by a Mathieu equation (see McLachlan, 1947, pp. 19-20 and 82-3). Thus,

$$
\frac{\mathrm{d}^{2} z}{\mathrm{~d} \phi^{2}}+z\left(a_{0}+2 a_{2} \cos 2 \phi\right)=0
$$

and in accordance with the theory of the Mathieu function, the frequency is given as

$$
v^{2}=a_{0}-\frac{a_{0}-1}{2\left(a_{0}-1\right)^{2}-a_{2}^{2}} a_{2}^{2}+\cdots
$$

whence the period becomes, using (41),

$$
P=\frac{2 \pi}{\sqrt{ } A}\left\{1-e^{2}\left[\frac{A-1}{4 A}\left(1-\frac{A-1}{4 A-1}\right)\right]+O\left(e^{4}\right)\right\}
$$


However, Equation (43) can give the motion, $z=z(\theta, e)$, correctly only to order $e A_{z}$ and not to the required $O\left(e^{2} A_{z}\right)$. It thus is necessary to solve Equation (39); Equation (45) then is a reference solution for comparison.

The solution is accomplished by means of the method of Lindsted and Poincaré, (Cole, 1965), which is also used to solve Equation (43). The method is one of analytic continuation with respect to a parameter, the solution being given formally as a power series in the parameter. The independent variable also is multiplied by a power series in the parameter, this series having undetermined coefficients. The original differential equation then is exhibited as a set of linear differential equations, ordered according to the powers of the parameter, which may be solved recursively; the undetermined coefficients are given uniquely by the condition that no secular terms (such as $t$ cos $\sqrt{A} t$ ) appear in the solution. The resulting solution is bounded (not necessarily periodic).

Thus, in Equation (39), we take

$$
\begin{aligned}
& z=A_{z}\left[z_{1}+e z_{2}+e^{2} z_{3}+\cdots\right] \\
& \theta=\tau\left[1+\delta_{2} e^{2}+O\left(e^{4}\right)\right]
\end{aligned}
$$

so that, to third order,

$$
\frac{\mathrm{d}^{2} z}{\mathrm{~d} \theta^{2}}=A_{z}\left[\frac{\mathrm{d}^{2} z_{1}}{\mathrm{~d} \tau^{2}}+e \frac{\mathrm{d}^{2} z_{2}}{\mathrm{~d} \tau^{2}}+e^{2}\left(\frac{\mathrm{d}^{2} z_{3}}{\mathrm{~d} \tau^{2}}-2 \delta_{2} \frac{\mathrm{d}^{2} z_{1}}{\mathrm{~d} \tau^{2}}\right)\right]+O\left(e^{3} A_{z}\right)
$$

and Equation (39) becomes

$$
\begin{aligned}
& \frac{\mathrm{d}^{2}}{\mathrm{~d} \tau^{2}}\left[z_{1}+e z_{2}+e^{2} z_{3}\right]=A z_{1}+e\left[A z_{2}-(A-1) z_{1} \cos \theta\right]+ \\
& \quad+e^{2}\left[A z_{3}-(A-1) z_{2} \cos \theta+(A-1) z_{1} \cos ^{2} \theta+2 \delta_{2} A z_{1}\right]+O\left(e^{3}\right)
\end{aligned}
$$

which is written as a sequence:

Order one: $\mathrm{d}^{2} z_{1} / \mathrm{d} \tau^{2}+A z_{1}=0$

Order two: $\quad \mathrm{d}^{2} z_{2} / \mathrm{d} \tau^{2}+A z_{2}=(A-1) z_{1} \cos \theta$

Order three: $\mathrm{d}^{2} z_{3} / \mathrm{d} \tau^{2}+A z_{3}=(A-1)\left[z_{2} \cos \theta-z_{1} \cos ^{2} \theta\right]-2 \delta_{2} A z_{1}$

and the ordering is justified by the appearance of $A_{z}$ in (46).

Equation (48) is identical with Equation (7), with the solution Equation (9), except that $\tau$, defined by Equation (47), replaces $t$. Equation (49) then is solved as a linear equation with forcing function; only the particular solution is needed. Thus, $z_{2}$ is found:

$$
z_{2}=\frac{A-1}{2}\left(\frac{\sin (\sqrt{A}+\theta / \tau) \tau}{A-(\sqrt{\bar{A}}+\theta / \tau)^{2}}+\frac{\sin (\sqrt{A}-\theta / \tau) \tau}{A-(\sqrt{ } \bar{A}-\theta / \tau)^{2}}\right)
$$

or, to third order,

$$
z_{2}=\frac{A-1}{2}\left(\frac{\sin (\sqrt{A}-\theta / \tau) \tau}{2 \sqrt{ } \bar{A}-1}-\frac{\sin (\sqrt{ } \bar{A}+\theta / \tau) \tau}{2 \sqrt{ } \bar{A}+1}\right)
$$


The requirements of third-order accuracy imply that $(\theta / \tau)$ must be retained in the argument of the sines, but, from Equation (47), $(\theta / \tau)$ may be taken as unity when appearing as a coefficient, since $z_{2}$ is of order $e A_{z}$.

Then, Equation (50) becomes

$$
\begin{aligned}
\frac{\mathrm{d}^{2} z_{3}}{\mathrm{~d} \tau^{2}}+A z_{3}= & \frac{(A-1)^{2}}{4}\left[\frac{\sin (\sqrt{A}-2 \theta / \tau) \tau}{2 \sqrt{A}-1}-\frac{\sin (\sqrt{A}+2 \theta / \tau) \tau}{2 \sqrt{A}+1}+\right. \\
& \left.+\sin \sqrt{A} \tau\left(\frac{1}{2 \sqrt{A}-1}-\frac{1}{2 \sqrt{A}+1}\right)\right]-\frac{A-1}{4} \times \\
& \times[\sin (\sqrt{ } \bar{A}+2 \theta / \tau) \tau+\sin (\sqrt{ } \bar{A}-2 \theta / \tau) \tau+ \\
& +2 \sin \sqrt{A} \tau]-2 \delta_{2} A \sin \sqrt{A} \tau
\end{aligned}
$$

The terms in $\sin \sqrt{A} \tau$ on the right-hand side of (53) are in resonance with the lefthand side; thus, their coefficients must sum to zero so as to avoid secular terms in the solution, and this requirement determines $\delta_{2}$ :

$$
\delta_{2}=\frac{A-1}{4 A}\left[\frac{A-1}{4 A-1}-1\right]
$$

in agreement with Equation (45). Equation (53) then is solved immediately:

$$
\begin{aligned}
z_{3}= & \frac{\sqrt{A}(A-1)}{4}\left[\frac{(\sqrt{A}-2) \sin (\sqrt{A}-2 \theta / \tau) \tau}{(2 \sqrt{A}-1)(4 \sqrt{A}-4)}+\right. \\
& \left.+\frac{(\sqrt{A}+2) \sin (\sqrt{A}+2 \theta / \tau) \tau}{(2 \sqrt{A}+1)(4 \sqrt{A}+4)}\right]
\end{aligned}
$$

The solution to Equation (39) then is given by Equations (46, 47); $z_{2}, z_{3}$ by Equations $(52,55)$ respectively; and $\delta_{2}$ by Equation $(54)$. This completes the third-order solution in the case of linear motion in the elliptic problem.

\section{Circular Problem - Nonlinear Motion}

In extending the results of the previous section to the case of finite amplitude, the pertinent equation is (38), which is to be treated as an extension of (11). Thus it is necessary to solve equations analogous to (13), which are found from (4):

$$
\begin{aligned}
& x^{\prime \prime}-2 y^{\prime}=(1-e \cos \theta)\left[(1+2 A) x+\frac{3}{2} B z^{2}\right] \\
& y^{\prime \prime}+2 x^{\prime}=(1-e \cos \theta)(1-A) y
\end{aligned}
$$

The solution to Equation (56) is of importance in exhibiting the complete threedimensional motion derived from the generating solution, Equation (9), but is not germane to the present discussion. It is therefore deferred to the Appendix; for this discussion, it is sufficient to note that the third-order solution will consist of two sets of terms, the first of order $A_{z}^{2}$ and the second of order $e A_{z}^{2}$. 
Equation (38) may be written as an extension of (11):

$$
z^{\prime \prime}+z(1+e \cos \theta)^{-1}\left[e \cos \theta+A-3 B x+\frac{3}{2}\left(4 x^{2}-y^{2}-z^{2}\right)+\cdots\right]
$$

In (57), $x$ need only be given to second order; thus, Equation (15) holds, and the notation of Equations (16-18) remains valid without change. Accordingly, Equation (57) becomes

$$
\mathrm{d}^{2} z / \mathrm{d} \theta^{2}+(1+e \cos \theta)^{-1}\left[u z-\frac{3}{2} v z^{3}+z e \cos \theta\right]=0
$$

which will give the additional terms (of order $e A_{z}^{2}$ ) needed for a complete third-order theory.

Again, Lindsted's method is employed. We assume an expansion of $z$ precisely as in Equations (46). In developing the expansion of Equation (58), we have

$$
\begin{aligned}
& \theta=\tau\left[1+\delta_{1} e+\delta_{2} e^{2}+O\left(e^{3}\right)\right] \\
& (1+e \cos \theta)^{-1}=1-e \cos \theta+e^{2} \cos ^{2} \theta-e^{3} \cos ^{3} \theta+\cdots \\
& z^{3}=z_{1}^{3}+e\left(3 z_{1}^{2} z_{2}\right)+e^{2}\left[3\left(z_{1} z_{2}^{2}+z_{3} z_{1}^{2}\right)\right]+O\left(e^{3}\right)
\end{aligned}
$$

Note that Equation (59) differs from Equation (47).

The solution to Equation (58) will involve terms of all orders in $e$ and $A_{z}$, but only terms up to order $e A_{z}^{2}$ are needed, the other terms needed for a third-order theory being already available. Thus (58) becomes

$$
\begin{aligned}
& \mathrm{d}^{2} z / \mathrm{d} \tau^{2}+\left[u z_{1}-\frac{3}{2} v z_{1}^{3}\right]+e[\left(2 \delta_{1}-\cos \theta\right)\left(u z_{1}-\frac{3}{2} v z_{1}^{3}\right)+u z_{2}- \\
&\left.-\frac{9}{2} v z_{1}^{2} z_{2}+z_{1} \cos \theta\right]+O\left(e^{2}\right)=0
\end{aligned}
$$

or

$$
\begin{aligned}
& \mathrm{d}^{2} z_{1} / \mathrm{d} \tau^{2}+u z_{1}-\frac{3}{2} v z_{1}^{3}=0 \\
& \mathrm{~d}^{2} z_{2} / \mathrm{d} \tau^{2}+\left(u-\frac{9}{2} v z_{1}^{2}\right) z_{2}=\left(\cos \theta-2 \delta_{1}\right)\left(u z_{1}-\frac{3}{2} v z_{1}^{3}\right)-z_{1} \cos \theta
\end{aligned}
$$

Equation (64) is simply Equation (16), with solution given by Equation (27), with $t$ replaced by $\tau$.

However, this is not the end of the matter; for the solution $z_{1}$ must be written in a form suitable for use in Equation (64). This may be done using the Fourier-series solution of Equation (16), which is Equation (37). Making use of Equations (30, 31) gives

$$
z_{1}=\sin \left[\left(1-\frac{9}{16} \frac{v}{u} A_{z}^{2}\right) \sqrt{u} \tau\right]+O\left(A_{z}^{2}\right)
$$

Now define a new independent variable, $\zeta$ :

$$
\zeta=\tau\left(1-\frac{9}{16} \frac{v}{u} A_{z}^{2}\right)
$$


so Equation (65) becomes

$$
z_{1}=\sin \sqrt{u} \zeta+O\left(A_{z}^{2}\right)
$$

The importance of this transformation is that use of $\zeta$ instead of $\tau$ will give true resonance of certain of the forcing terms on the righthand side of Equation (64) with the natural frequency associated with the left-hand side, i.e. the resonance will not merely be 'approximate' (correct to within a term of order $A_{z}^{2}$ ). This is required by the Lindsted method.

In (64), perform a change of independent variable: $\tau$ is replaced by $\zeta$. Then,

$$
\begin{aligned}
\mathrm{d}^{2} z_{2} / \mathrm{d} \zeta^{2} & +\left(1+\frac{9}{8} \frac{v}{u} A_{z}^{2}\right)\left(u-\frac{9}{2} v z_{1}^{2}\right) z_{2} \\
& =\left(1+\frac{9}{8} \frac{v}{u} A_{z}^{2}\right)\left[\left(\cos \theta-2 \delta_{1}\right)\left(u z_{1}-\frac{3}{2} v z_{1}^{3}\right)-z_{1} \cos \theta\right]
\end{aligned}
$$

Again we apply Lindsted's method, but now the perturbation parameter is $A_{z}$ and not $e$, so that the independent variable has been scaled twice: once with $e$, then again with $A_{z}$, as the perturbation parameter.

To solve (68), we have

$$
\begin{aligned}
& z_{1}=z_{01}+A_{z}^{2} z_{03}+\cdots \\
& z_{2}=z_{11}+A_{z} z_{12}+\cdots \\
& \delta_{1}=\delta_{10}+A_{z} \delta_{11}+\cdots
\end{aligned}
$$

where the subscript convention serves to fix the position of each term in a power series expansion of $z=z(\zeta)$ in the parameters $e, A_{z}$. The first subscript gives the order of $e$, the second of $A_{z}$; thus, $z_{12}$ is of order $e A_{z}^{2}$, and $z_{1}, z_{2}$ were defined in Equation (46). Note that $z_{1}$ is given by Equation (67) and that from (47), $\delta_{10}=0$. Then, expanding (68) and ordering the terms, we have

$$
\begin{aligned}
& \mathrm{d}^{2} z_{11} / \mathrm{d} \zeta^{2}+u z_{11}=(u-1) \cos \theta \sin \sqrt{u} \zeta \\
& \mathrm{d}^{2} z_{12} / \mathrm{d} \zeta^{2}+u z_{12}=-2 \delta_{11} u \cdot \sin \sqrt{u} \zeta
\end{aligned}
$$

Equation (70) is simply Equation (49), with $u$ replacing $A$ and $\zeta$ replacing $\tau$. The solution, Equation (52), has been given.

Equation (71) is even simpler; the avoidance of secularity requires $\delta_{11}=0$. Then $z_{12}=0$.

The remarkable conclusion is that the study of nonlinear motion in the elliptic problem contributes no new terms to the formal third-order expressions for the motion. The terms representing nonlinearity and eccentricity effects may be computed separately and added; the effects on the period of motion may be computed separately and multiplied. 


\section{Summary}

The third-order out-of-plane motion is given by Equations (27), (52), (55) as modified in accordance with Equation (62):

$$
\begin{aligned}
z= & A_{z} \operatorname{sn}\left[\zeta \sqrt{u}\left(1+\frac{3}{16} \frac{v}{u} A_{z}^{2}\right), \frac{1}{2} A_{z} \sqrt{3 v / u}\right] \\
& +e A_{z} \cdot \frac{A-1}{2}\left[\frac{\sin (\sqrt{u}-\theta / \zeta) \zeta}{2 \sqrt{A}-1}-\frac{\sin (\sqrt{u}+\theta / \zeta) \zeta}{2 \sqrt{A}+1}\right]+ \\
& +e^{2} A_{z} \cdot \frac{\sqrt{A}(A-1)}{4}\left[\frac{(\sqrt{A}-2) \sin (\sqrt{u}-2 \theta / \zeta) \zeta}{(2 \sqrt{A}-1)(4 \sqrt{A}-4)}\right. \\
& \left.+\frac{(\sqrt{A}+2) \sin (\sqrt{u}+2 \theta / \zeta) \zeta}{(2 \sqrt{\bar{A}}+1)(4 \sqrt{\bar{A}}+4)}\right],
\end{aligned}
$$

where, from Equations $(66,54,47)$,

$$
\theta=\zeta\left(1-\frac{3}{4} \frac{A-1}{4 A-1} e^{2}\right)\left(1+\frac{9}{16} \frac{v}{A} A_{z}^{2}\right)
$$

and in Equations $(72,73)$ there is taken $u=A, \zeta=\theta$ where such simplifications will not detract from third-order accuracy. Since $A>1$ (see Szebehely (1967), pp. 214-5), Equation (73) shows that eccentricity acts to decrease the period, nonlinearity to increase it. However, the motion is not periodic, since Equation (72) contains terms which are not of period $\sqrt{ } u$ or its multiples. The motion, neglecting eccentricity, is periodic, however, and the eccentricity may be considered to add additional terms of noncommensurable periodicity.

The third-order in-plane motion is given in the Appendix, as the solution of Equation (56).

\section{Triangular Points}

Having derived a third-order theory for the collinear points, we now turn our attention to the triangular points. The case of the triangular points. is studied in the same manner as is the case of the collinear points. Equations of motion, analogous to $(10,11)$, are given by expansions of Equations (1) about $\left(x_{0}, y_{0}, 0\right)=$ $=\left(\frac{1}{2}-\mu, \pm \frac{1}{2} \sqrt{ } 3,0\right)$. We restrict ourselves to the case of the $L_{4}$ point, with the understanding that results for the $L_{5}$ point may be obtained by changing the sign of $\sqrt{ } 3$ wherever it appears. Accordingly,

$$
\begin{aligned}
\ddot{x}-2 \dot{y}= & \frac{3}{4} x+\frac{3 \sqrt{ } 3}{4}(1-2 \mu) y+ \\
& +\frac{21}{16}(1-2 \mu) x^{2}-\frac{3 \sqrt{ } 3}{8} x y-\frac{33}{16}(1-2 \mu) y^{2}+\frac{3}{4}(1-2 \mu) z^{2}+ \\
& +\cdots
\end{aligned}
$$




$$
\begin{aligned}
\ddot{y}-2 \dot{x}= & \frac{3 \sqrt{ } 3}{4}(1-2 \mu) x+\frac{9}{4} y- \\
& -\frac{3 \sqrt{ } 3}{16} x^{2}-\frac{33}{8}(1-2 \mu) x y-\frac{9 \sqrt{ } 3}{16} y^{2}+\frac{3 \sqrt{ } 3}{4} z^{2}+ \\
& +\cdots \\
\ddot{z}+z= & \frac{3}{2}(1-2 \mu) x z+\frac{3 \sqrt{ } 3}{2} y z- \\
& -\frac{3}{8} x^{2} z-\frac{33}{8} y^{2} z+\frac{3}{2} z^{3}-\frac{15 \sqrt{ } 3}{4}(1-2 \mu) x y z+ \\
& +\cdots
\end{aligned}
$$

The linearized solution for out-of-plane motion is given:

$$
z=A_{z} \sin t
$$

and, since $z \gg x, y$, Equations $(74,75)$ may be simplified to yield an analog to Equation (13):

$$
\begin{aligned}
& \ddot{x}-2 \dot{y}-\frac{3}{4} x-\frac{3 \sqrt{ } 3}{4}(1-2 \mu) y=\frac{3}{4}(1-2 \mu) z^{2} \\
& \ddot{y}+2 \dot{x}-\frac{3 \sqrt{ } 3}{4}(1-2 \mu) x-\frac{9}{4} y=\frac{3 \sqrt{ } 3}{4} z^{2}
\end{aligned}
$$

and

$$
z^{2}=\frac{1}{2}(1-\cos 2 t) A_{z}^{2}
$$

and since Equations (78) are linear and inhomogeneous with forcing functions of frequency 0 and 2, from Equation (79), it follows that the particular solution of (78) has the forms,

$$
\begin{aligned}
& x=C_{0}+C_{2} \cos 2 t+S_{2}^{\prime} \sin 2 t \\
& y=C_{0}^{\prime}+S_{2} \sin 2 t+C_{2}^{\prime} \cos 2 t
\end{aligned}
$$

where the coefficients are given by

$$
\begin{aligned}
& {\left[\begin{array}{l}
C_{0} \\
C_{0}^{\prime}
\end{array}\right] }=\frac{16 A_{z}^{2}}{27\left[(1-2 \mu)^{2}-1\right]} \times \\
& \times\left[\begin{array}{cc}
\frac{9}{4} & -\frac{3 \sqrt{3}}{4}(1-2 \mu) \\
-\frac{3 \sqrt{3}}{4}(1-2 \mu) & \frac{3}{4}
\end{array}\right]\left[\begin{array}{c}
\frac{3}{8}(1-2 \mu) \\
\frac{3 \sqrt{3}}{8}
\end{array}\right] \\
& {\left[\begin{array}{l}
C_{2} \\
S_{2}^{\prime} \\
S_{2} \\
C_{2}^{\prime}
\end{array}\right]=\frac{4 A_{z}^{2}}{3\left[9(1-2 \mu)^{2}-73\right]} \times }
\end{aligned}
$$




$$
\begin{aligned}
& \times\left[\begin{array}{cccc}
25 & 0 & -16 & -3 \sqrt{3}(1-2 \mu) \\
0 & 25 & -3 \sqrt{3}(1-2 \mu) & 16 \\
-16 & -3 \sqrt{3}(1-2 \mu) & 19 & 0 \\
-3 \sqrt{3}(1-2 \mu) & 16 & 0 & 19
\end{array}\right] \times \\
& \times\left[\begin{array}{c}
\frac{3}{8}(1-2 \mu) \\
0 \\
0 \\
\left.\frac{3 \sqrt{3}}{8}\right]
\end{array}\right]
\end{aligned}
$$

as may be seen by directly substituting Equations $(79,80)$ into $(78)$ and solving for the coefficients. Thus, Equations (80) become

$$
\begin{aligned}
& x=\frac{8 A_{z}^{2}}{73-9(1-2 \mu)^{2}}[(1-2 \mu) \cos 2 t+\sqrt{3} \sin 2 t] \\
& y=-\frac{\sqrt{\varepsilon}}{6} A_{z}^{2}+\frac{A_{z}^{2}}{73-9(1-2 \mu)^{2}} \times \\
& \quad \times\left[\frac{\sqrt{3}}{2}\left[19-3(1-2 \mu)^{2}\right] \cos 2 t-8(1-2 \mu) \sin 2 t\right]
\end{aligned}
$$

Now, in (76), we retain terms only up to order $A_{z}^{3}$ and obtain

$$
\ddot{z}+z-\frac{3}{2}[(1-2 \mu) x+\sqrt{3} y] z-\frac{3}{2} z^{3}=0
$$

and from $(81,82)$,

$$
(1-2 \mu) x+\sqrt{3} y=\frac{1}{2}\left[\frac{57+7(1-2 \mu)^{2}}{73-9(1-2 \mu)^{2}} \cos 2 t-1\right] A_{z}^{2}
$$

Note that in (84), there is cancellation of terms in $\sin 2 t$. It is this cancellation which permits the expression of (84) in the form of Equation (16), permitting solution by elliptic functions. Thus, from (77),

$$
\begin{aligned}
& \sin t=z / A_{z} \\
& \sin t \cos 2 t=z / A_{z}-2 z^{3} / A_{z}^{3}
\end{aligned}
$$

so that (83) becomes

$$
\ddot{z}+z\left[1+\frac{48 \mu(1-\mu)}{73-9(1-2 \mu)^{2}} A_{z}^{2}\right]-\frac{3}{2} z^{3}\left[\frac{64 \mu(1-\mu)}{73-9(1-2 \mu)^{2}}\right]=0
$$

or

$$
\ddot{z}+u z-\frac{3}{2} v z^{3}=0
$$

where $(86,16)$ are identical. The solution then follows immediately from the equations given for the collinear-point solutions. In particular, there is:

The first integral, Equations $(19,20)$ 
The formal solution, Equations $(27,28)$

The period of motion, Equation (29)

The Fourier-series expansion, Equations (33-37)

To write these explicitly, define

$$
\gamma \equiv \frac{16 \mu(1-\mu)}{73-9(1-2 \mu)^{2}}
$$

Then we have:

The first integral:

$$
I=A_{z}^{2}
$$

The formal solution:

$$
z=A_{z} \operatorname{sn}\left(t, A_{z} \sqrt{3 \gamma}\right)
$$

The period of motion:

$$
P=2 \pi\left(1+\frac{3}{4} \gamma A_{z}^{2}\right)
$$

The Fourier-series expansion:

$$
z=A_{z} \sin \tau+\frac{3}{16} \gamma A_{z}^{3}(\sin \tau+\sin 3 \tau)+O\left(A_{z}^{5}\right)
$$

where

$$
t=\tau\left(1+\frac{3}{4} \gamma A_{z}^{2}\right)
$$

and $A_{z}$ is given by Equation (88). The solution due to Buck (1920) involves initial conditions selected so that the amplitude $A_{z}$ is unchanged due to incorporation of nonlinear terms in Equation (91); the resulting solution gives for the terms in $A_{z}^{3}$ in Equation (91), $(\sin 3 \tau-3 \sin \tau)$. The present solution involves no such special initial conditions. When this initial-conditions effect is allowed for, Equation (91) becomes identical with the solution of Buck.

It is clear that $\gamma$ is a monotone increasing function of $\mu$, reaching its maximum value of $\gamma=4 / 73$ at $\mu=\frac{1}{2}$. For the small values of $\mu$ which are of practical interest,

$$
\gamma \sim \frac{\mu}{64}(16-25 \mu)
$$

which is accurate to order $\mu^{3}$.

\section{Relation to Existing Solutions}

References to existing work on out-of-plane motion include Moulton (1920), Farquhar and Kamel (1972) for the collinear points, and Buck (1920) for the triangular points. Farquhar and Kamel (1972) gives general third-order expansions for three-dimensional motion about the Earth-Moon $L_{2}$ point. In principle, the coefficients of Equation 
(72) may be evaluated for this case, and the result should be equivalent to a special case of Farquhar and Kamel. However, the expansions of Farquhar and Kamel are given with independent variable $t$ (not $\theta$ ). Thus, these coefficients will be entirely different from those of Farquhar and Kamel, so that no useful comparison can be made.

The elliptic-function solutions were shown to be equivalent to the solutions of Moulton (1920) and Buck (1920). Thus, for plots or diagrams of these solutions, see respectively Moulton (1920), p. 176, and Buck (1920), p. 323. The collinear-point solutions are also depicted in Szebehely (1967), p. 575.

\section{Appendix: Solution to Equation (56)}

A solution to Equation (56) is required to give the third-order expressions for the in-plane motion associated with the out-of-plane solution for the collinear points. Because of the linearity of the equations, it is not necessary to apply Lindsted's method; the solution involves merely the solution of inhomogeneous linear differential equations.

Such solutions involve equations of the form

$$
\begin{aligned}
& x^{\prime \prime}-2 y^{\prime}-(1+2 A) x=a_{\omega} \cos \omega \theta \\
& y^{\prime \prime}+2 x^{\prime}-(1-A) y=b_{\omega} \sin \omega \theta
\end{aligned}
$$

with solution

and

$$
x=c_{\omega} \cos \omega \theta, \quad y=d_{\omega} \sin \omega \theta
$$

$\left[\begin{array}{c}c_{\omega} \\ d_{\omega}\end{array}\right]+\frac{\left[\begin{array}{cc}\omega^{2}-(1-A) & -2 \omega \\ -2 \omega & \omega^{2}+(1+2 A)\end{array}\right]}{\omega^{4}-[4-(1+2 A)-(1-A)] \omega^{2}+(1-A)(1+2 A)}\left[\begin{array}{l}a_{\omega} \\ b_{\omega}\end{array}\right]=\left[\begin{array}{l}0 \\ 0\end{array}\right]$

Equation (95) is derived from $(93,94)$. The use of the subscript ()$_{w}$ indicates the term under discussion; thus, $a_{2}$ would refer to a forcing term in $\cos 2 \theta, d_{\sqrt{A}}$ would refer to a solution term in $\sin \sqrt{A} \theta$. Also, the matrix of coefficients in (95) is denoted $\underline{\underline{M_{\omega}}}$; thus, (95) is written,

$$
\left[\begin{array}{ll}
c_{\omega} & d_{\omega}
\end{array}\right]^{T}+M_{\omega}\left[\begin{array}{ll}
\alpha_{\omega} & b_{\omega}
\end{array}\right]^{T}=\left[\begin{array}{ll}
0 & 0
\end{array}\right]^{T}
$$

where []$^{T}$ indicates the transpose.

Consider Equation (56). The solution will have the form

$$
x=x_{2}+e x_{3} ; \quad y=y_{2}+e y_{3}
$$

where the subscript indicates the order of the term. In addition, a second-order expression for $z$ is required, and is found from $(67,72)$ :

$$
z=A_{z} \sin \sqrt{u} \zeta+e A_{z} \cdot \frac{A-1}{2}\left[\frac{\sin (\sqrt{u}-\theta / \zeta) \zeta}{2 \sqrt{A}-1}-\frac{\sin (\sqrt{ } \bar{u}+\theta / \zeta) \zeta}{2 \sqrt{A}+1}\right]
$$


or

$$
z=z_{1}+e z_{2}
$$

Combining Equations $(97,99)$ with $(56)$ and separating terms gives the equations for the in-plane motion:

$\operatorname{Order} A_{z}^{2}: x_{2}^{\prime \prime}-2 y_{2}^{\prime}-(1+2 A) x_{2}=\frac{3}{2} B z_{1}^{2}$

$$
y_{2}^{\prime \prime}+2 x_{2}^{\prime}-(1-A) y_{2}=0
$$

Order $e A_{z}^{2}: x_{3}^{\prime \prime}-2 y_{3}^{\prime}-(1+2 A) x_{3}=-(1+2 A) x_{2} \cos \theta+$

$$
\begin{aligned}
& +\frac{3}{2} B\left(2 z_{1} z_{2}-z_{1}^{2} \cos \theta\right) \\
y_{3}^{\prime \prime}+2 x_{3}^{\prime}-(1-A) y_{3}= & -(1-A) y_{2} \cos \theta
\end{aligned}
$$

and there are no terms of order $A_{z} e^{2}$. Equation (100) is simply Equation (13); the solution is (14), except that the trigonometric terms are of argument $2 \sqrt{u} \zeta$ instead of $2 \sqrt{A} t$.

Thus, the terms $z_{1}, z_{2}, x_{2}, y_{2}$ are known, and the right-hand sides of Equation (101) may be constructed. Denoted, respectively, $R H S_{x}$ and $R H S_{y}$, they are

$$
\begin{aligned}
R H S_{x} & =\left[\frac{1}{2}+\frac{3}{4} B\left(\frac{A-1}{4 A-1}+\frac{2}{1+2 A}\right)\right] \cos \theta- \\
& -\left[\frac{1}{4}+\frac{3}{4} B\left(\frac{(1+2 A)(1+3 A)}{2\left(1-7 A+18 A^{2}\right)}-\frac{A-1}{2 \sqrt{A}-1}\right)\right] \cos (2 \sqrt{u}+\theta / \zeta) \zeta- \\
& -\left[\frac{1}{4}+\frac{3}{4} B\left(\frac{(1+2 A)(1+3 A)}{2\left(1-7 A+18 A^{2}\right)}+\frac{A-1}{2 \sqrt{A}-1}\right)\right] \cos (2 \sqrt{u}-\theta / \zeta) \zeta \\
R H S_{y} & =\frac{3}{2} \frac{B \sqrt{A}(1-A)}{1-7 A+18 A^{2}}[\cos (2 \sqrt{u}-\theta / \zeta) \zeta-\cos (2 \sqrt{u}+\theta / \zeta) \zeta]
\end{aligned}
$$

There are thus three frequencies: $\omega=1, \omega=\sqrt{u} \pm \theta / \zeta$. However, for third-order accuracy the last two frequencies may be taken as $\omega=\sqrt{ } A \pm 1$. The solution then proceeds: From (102), the terms $a_{1}, b_{1} ; a_{\sqrt{A}+1}, b_{\sqrt{A}+1} ; a_{\sqrt{A}-1}, b_{\sqrt{A}-1}$ are read off by comparison with (93); the matrices $\underline{\underline{M}}_{1}, \underline{\underline{M}}_{\sqrt{A}+1}, \underline{\underline{M}}_{\sqrt{A}-1}$ are computed respectively from $(95,96)$; and the coefficients $c_{1}, d_{1} ; c_{\sqrt{A}+1}, \overline{d_{\sqrt{A}+1}} ; c_{\sqrt{A}-1}, d_{\sqrt{A}-1}$ are computed directly from (95). Note that $b_{1}=0$.

Then $z_{3}, y_{3}$ are written

$$
\begin{aligned}
& x_{3}=c_{1} \cos \theta+c_{\sqrt{A}+1} \cos (\sqrt{u}+\theta / \zeta) \zeta+c_{\sqrt{A}-1} \cos (\sqrt{u}-\theta / \zeta) \zeta \\
& y_{3}=d_{1} \sin \theta+d_{\sqrt{A}+1} \sin (\sqrt{ } \bar{u}+\theta / \zeta) \zeta+d_{\sqrt{A}-1} \sin (\sqrt{u}+\theta / \zeta) \zeta
\end{aligned}
$$

Note that the $c$ 's and $d$ 's are all of order $A_{z}^{2}$. The solution to third order in $e$ and $A_{z}$ for out-of-plane motion about collinear points is thus complete. 


\section{References}

Buck, T.: 1920, 'Oscillating Satellites Near the Lagrangian Equilateral-Triangular Points', in F. R. Moulton (ed.), Periodic Orbits, Carnegie Institute of Washington, pp. 300-324.

Cole, J. D.: 1965, Perturbation Methods in Applied Mathematics, Blaisdell, Waltham, Mass., pp. 79-102.

Davis, H. T.: 1962, Introduction to Nonlinear Differential and Integral Equations, Dover, New York. Farquhar, R. W. and Kamel, A. A.: 1972, 'Quasi-Periodic Orbits about the Translunar Libration Point', AIAA Paper 72-935, AIAA/AAS Astrodynamics Conf., Sept. 1972. (Solutions previously listed in NASA TN D-6365, July 1971.)

Goldstein, H.: 1965, Classical Mechanics, Addison-Wesley, Reading, Mass.

Heppenheimer, T. A.: 1970, 'Optimal Controls for Out-of-Plane Motion about the Translunar Libration Point', J. Spacecraft Rockets 7, 1088-1092.

Mathews, J. and Walker, R. L.: 1964, Mathematical Methods of Physics, W. A. Benjamin, New York. McLachlan, N. W.: 1947, Theory and Applications of Mathieu Functions, Clarendon Press, Oxford. Moulton, F. R.: 1920, Periodic Orbits, Carnegie Institute of Washington, pp. 151-180.

Szebehely, V.: 1967, Theory of Orbits, Academic Press, New York.

Whittaker, E. T. and Watson, G. N.: 1963, A Course of Modern Analysis, Cambridge University Press. 